\title{
Recuperação ativa favorece a retirada simpática pós-exercício máximo em jovens não treinadas
}

http://dx.doi.org/10.11606/1807-5509201900030353

\author{
Jaqueline Alves de ARAÚJO* \\ Gabriel Kolesny TRICOT* \\ Gisela ARSA* \\ Lucieli Teresa CAMBRI*
}

*Departamento de Educação Física Universidade Federal de Mato Grosso, Cuiabá, MT, Brasil.

\section{Resumo}

0 objetivo deste estudo foi avaliar a variabilidade da frequência cardiaca (VFC) após recuperação ativa de um exercício máximo em jovens não treinadas. Nove mulheres eutróficas $(25,45 \pm 5,41$ anos; $22,23 \pm$ $2,03 \mathrm{~kg} \cdot \mathrm{m}^{-2} ; 28,13 \pm 5,0 \%$ de gordura corporal) foram avaliadas em repouso, exercício e na recuperação pós teste progressivo máximo em cicloergômetro realizada de forma ativa $(A)$ ou passiva $(P)$, com os indices da VFC (RMSSD e SDNN) avaliados durante os 5 min da recuperação e nos 5 min posteriores, janelados a cada $30 \mathrm{~s}$. Os índices $\operatorname{LnRMSSD}_{30 \mathrm{~s}}$ e $\operatorname{LnSDNN}_{30 \mathrm{~s}}$ ao final do exercício $(A: 0,52 \pm 0,13 ; \mathrm{P}: 0,55$ $\pm 0,25$ / A: $0,42 \pm 0,11 ; P: 0,48 \pm 0,21 \mathrm{~ms}$ ) reduziram em relação ao repouso (A: $1,49 \pm 0,29 ; P: 1,46 \pm$ $0,28 / A: 1,70 \pm 0,21 ; P: 1,69 \pm 0,20 \mathrm{~ms})$ e permaneceram reduzidos $(p<0,05)$ ao final dos primeiros 5 min da recuperação (A: $0,53 \pm 0,12 ; P: 0,45 \pm 0,14$ / A: 0,63 $\pm 0,12 ; P: 0,68 \pm 0,24 \mathrm{~ms})$ e após esse período ( $A: 0,48 \pm 0,12 ; P: 0,55 \pm 0,23 / A: 0,86 \pm 0,20 ; P: 0,87 \pm 0,20 \mathrm{~ms})$, sem diferença entre os tipos de recuperação. Entretanto, após a recuperação ativa, o índice $\operatorname{LnSDNN}_{30 \mathrm{~s}}$ elevou-se em relação ao final do exercício $(p<0,05)$. Em conclusão, independentemente do tipo de recuperação, não houve reativação vagal nos 10 min após um exercício máximo em mulheres jovens não treinadas, contudo a recuperação ativa auxiliou no restabelecimento da modulação autonômica cardíaca pela retirada da atividade simpática.

Palavras-chave: Sistema Nervoso Autonômico; Variabilidade da Frequência Cardíaca; Reativação Vagal.

\section{Introdução}

O exercício físico é um estimulo estressor que provoca alterações na frequência cardíaca $(\mathrm{FC})$ e na sua variabilidade (VFC) no período pós-exercício ${ }^{1,2}$ devido a reduzida atividade parassimpática, causando maior exposição à riscos cardiovasculares ${ }^{3}$. Nos instantes iniciais após o exercício submáximo, a $\mathrm{FC}$ diminui rapidamente devido à reativação vagal, e posteriormente, devido à retirada da atividade nervosa simpática ${ }^{4-6}$. Em contrapartida, pós-exercício máximo a retirada simpática parece ser a principal responsável pelo restabelecimento da modulação autonômica cardíaca ${ }^{7}$.

A partir disso, além da avaliação de diferentes populações, sobretudo em homens, e protocolos de exercício ${ }^{7-12}$, os ajustes do sistema nervoso autonômico ${ }^{13,14}$ são analisados após distintos tipos de recuperação, como a passiva ${ }^{5-7,11,15}$, ativa ${ }^{13}$, e em menor escala a comparação entre os tipos de recuperação ${ }^{14,16}$. O período de tempo analisado na recuperação varia entre três e $10 \min ^{2,7,10,11,17}$ até 20 a $83 \min ^{2,5,14,16,18}$. Assim, o tipo e a duração da recuperação pós-exercício provocam distintos ajustes na modulação autonômica cardíaca ${ }^{8-10,19} \mathrm{e}$ são amplamente analisados pelo comportamento da $\mathrm{FC}^{8,9,12,19}$, sem considerar a interação simpatovagal $^{1,7,11}$. Desta forma, a análise dos índices de VFC no domínio do tempo, especialmente o RMSSD e o SDNN, que, respectivamente, refletem a atuação da atividade parassimpática e a interação da atividade simpática e parassimpática, é um método validado a partir da utilização do bloqueador parassimpático atropina ${ }^{10}$.

Contudo, a influência do tipo de recuperação no restabelecimento da modulação autonômica 
cardíaca não está completamente elucidada. Uma vez que a recuperação ativa pós-exercício submáximo a $60 \%$ da FC máxima causou menor redução da $\mathrm{FC}^{19}$ e após exercício submáximo a $60-70 \%$ da FC de reserva não influenciou no balanço autonômico cardíaco em relação à recuperação passiva avaliada durante $30 \mathrm{~min}^{14}$, mesmo pós-exercício máximo com a recuperação analisada por $90 \mathrm{~min}^{16}$, embora os índices da VFC não tenham sido avaliados a partir de curtos janelamentos que é o mais adequado ${ }^{10}$.

Diante disso, são necessários estudos que avaliem a influência do tipo de recuperação pela análise dos índices da VFC pós-exercício máximo, especialmente em jovens mulheres não treinadas, uma vez que, após exercício máximo não foi encontrada reativação vagal, além disso,

\section{Método}

\section{Participantes}

Foram avaliadas nove mulheres jovens $(25,45 \pm$ 5,41 anos) eutróficas (massa corporal: 57,21 \pm 7,68 $\mathrm{kg}$; estatura: 1,60 $\pm 0,07 \mathrm{~m}$; índice de massa corporal: $22,23 \pm 2,03 \mathrm{~kg} \cdot \mathrm{m}^{-2}$; circunferência da cintura: 70,61 $\pm 5,99$ e $28,13 \pm 5,00 \%$ de gordura corporal), saudáveis, normotensas (pressão arterial sistólica: $105,11 \pm 7,62$; pressão arterial diastólica: 66,97 \pm $6,17 \mathrm{mmHg}$ ) e náo treinadas, por não praticarem exercício físico regular nos quatro meses anteriores ao início do estudo, a partir de amostragem não aleatória por conveniência. Os critérios de exclusão foram: fumar, uso de medicação contínua, doenças pré-existentes, morte súbita de familiares de primeiro grau com menos de 40 anos, problemas osteoarticulares, estar grávida ou na primeira semana da fase folicular do ciclo menstrual.

Todos os procedimentos do estudo foram aprovados pelo Comitê de Ética em Pesquisa da instituição ( $n^{\circ}$ 19109213.2.0000.5541) e todas as voluntárias assinaram um Termo de Consentimento Livre Esclarecido. Todas as coletas de dados ocorreram no período vespertino entre 15 e 19 hs, em uma sala silenciosa com temperatura média de $24,44 \pm 0,94^{\circ} \mathrm{C}$ e umidade relativa do ar de 43,11 $\pm 5,03 \%$, sem diferença entre as visitas.

\section{Procedimentos}

Duas visitas ao laboratório, com intervalo foi demonstrado que a recuperação ativa reduz riscos cardiovasculares, uma vez que a ação da bomba muscular não é subitamente interrompida contribuindo no retorno venoso e na remoção de metabólitos no pós-exercício ${ }^{16,19,20}$. Nesse sentido, a hipótese deste estudo é que não há reativação vagal nos primeiros $10 \mathrm{~min}$ subsequentes ao exercício máximo em mulheres jovens não treinadas, independentemente do tipo de recuperação, e, além disso, a janela de exposição cardiovascular será menor após a recuperação ativa, devido ao favorecimento de uma maior retirada simpática.

Portanto, o presente estudo teve como objetivo avaliar o comportamento da modulação autonômica cardíaca após recuperação ativa de exercício máximo em jovens não treinadas.

mínimo de 72 horas foram realizadas, para avaliações em repouso, exercício e recuperação pós-exercício.

Em ambos os dias, as voluntárias foram aconselhadas a não praticar atividades físicas vigorosas, e a não ingerir bebidas estimulantes nas 24 horas anteriores às avaliações. Adicionalmente, um lanche padronizado $(360,7 \mathrm{Kcal})$, composto por $73,19 \%$ de carboidratos $(66 \mathrm{~g}), 20,71 \%$ de lipídios $(8,3 \mathrm{~g})$ e $6,10 \%$ de proteínas $(5,5 \mathrm{~g})^{21}$, foi entregue as participantes para ingesta duas horas antes do ínicio das avaliaçōes.

$\mathrm{Na}$ primeira visita foi aplicada uma anamnese, composta por dados de identificação, relato de doenças e de histórico familiar. Se porventura a voluntária relatasse qualquer evento que pudesse oferecer risco à sua integridade durante as avaliações, seria excluída do estudo, porém, isto não ocorreu.

As variáveis de composição corporal coletadas foram: massa corporal (balança CAMRY ${ }^{\circledR}$ ) e estatura (estadiômetro $S A N N Y^{\circledR}$ ) para determinação do índice de massa corporal (IMC); medidas de circunferência da cintura (fita métrica CARDIOMED $^{\circledR}$ ). Além disso, foi determinado o percentual de gordura corporal por bioimpedância tetrapolar (MALTRON, BF 907).

Em seguida, as voluntárias adotaram a posição sentada em cadeira estofada em repouso por 15 min para realização de duas aferiçôes da pressão arterial (PA) (esfigmomanômetro semiautomático 
MICROLIFE BP3T0-A), com dois min de intervalo entre cada mensuração. Quando encontrada diferença de $5 \mathrm{mmHg}$ entre as mensuraçōes foi realizada uma terceira mensuração e calculada a média entre as duas aferiçôes com valores mais próximos, assim como, foi realizada a média dos valores dos dois dias de avaliaçôes. Nenhuma participante apresentou pressão arterial elevada $(>140 / 90 \mathrm{mmHg})^{22}$.

A FC e a VFC foram mensuradas utilizando um monitor de FC portátil (POLAR ${ }^{\circledR}$, modelo RS800CX) com registros batimento a batimento, por meio dos intervalos $\mathrm{R}-\mathrm{R}$, com a média dos 10 min finais de repouso considerada para análise. Os intervalos $\mathrm{R}-\mathrm{R}$ foram tratados no programa Kubios $H_{R V}{ }^{\circledR}$, com os artefatos filtrados no nível moderado.

Os índices da VFC foram determinados a partir de métodos não lineares no domínio do tempo: raiz quadrada da média das diferenças sucessivas ao quadrado, entre intervalos $\mathrm{R}-\mathrm{R}$ adjacentes (RMSSD), e o desvio padrão de todos os intervalos $\mathrm{R}-\mathrm{R}$ normais gravados em um intervalo de tempo (SDNN).

$\mathrm{Na}$ sequência, foi realizado um teste progressivo máximo em cicloergômetro (INBRAMED, CG$04)$, com carga inicial de $30 \mathrm{~W}$ e incrementos de 15 W a cada min, sendo orientado a manutenção de 60 rotações por min $(\mathrm{rpm})^{23}$. O teste foi encerrado pela ocorrência de exaustão voluntária máxima. Quando o último estágio não foi completado, a carga máxima foi determinada pela equação: Carga $_{\text {máx }}=$ Carga no estágio completo $+[$ (tempo (s) no último estágio/60) x 15 $]^{24}$. A percepção subjetiva do esforço (PSE) foi monitorada nos $10 \mathrm{~s}$ finais de cada estágio, a partir da escala de Borg $^{25}$ de 15 pontos. Ao final do teste progressivo foram obtidas a FC pico e a VFC pico. Além disso, durante o teste, o consumo oxigênio $\left(\mathrm{VO}_{2}\right)$ foi mensurado por meio da captação direta dos gases expirados por um analisador de gases (VO2000 MEDICAL GRAPHICS, INBRASPORT) e o maior valor obtido nos estágios finais do teste foi considerado como $\mathrm{VO}_{2}$ pico $^{26}$.

O teste foi considerado máximo em ambas as visitas, pois todas as voluntárias atingiram três dos seguintes critérios: incapacidade de manter as $60 \mathrm{rpm}$ por mais de $10 \mathrm{~s}$, no mínimo $90 \%$ da $\mathrm{FC}_{\text {máx }}$ predita pela idade (A: 99,19 \pm 4,46; P: 99, $14 \pm 4,56 \%)$, valores na escala de Borg > 18 pontos (A: 19,21 $\pm 2,32$; P: 19,56 $\pm 1,01)$ e quociente respiratório > $1.1(\mathrm{~A}: 1,19 \pm 0,05 ; \mathrm{P}$ : $1,16 \pm 0,07)$.

Ao final do teste progressivo, todas as avaliadas realizaram o período de recuperação de forma aleatória (ativa - A e passiva - P), por sorteio prévio, de forma ativa com $15 \mathrm{~W}$ ou passiva durante os cinco primeiros min, e posteriormente, mais cinco min sentadas no cicloergômetro em ambas as situaçôes. A FC e os índices da VFC na recuperação foram determinados em intervalos de $30 \mathrm{~s}$, durante os $10 \mathrm{~min} \mathrm{da}$ recuperação. Posteriormente os índices da VFC foram transformados em logaritmos naturais $\left(\operatorname{LnRMSSD}_{30 \mathrm{~s}} ; \operatorname{LnSDNN}_{30 \mathrm{~s}}\right)$ para permitir análise paramétrica ${ }^{10}$.

Foram determinadas as áreas sob a curva dos índices LnRMSSD ${ }_{30 s}$ e $\operatorname{LnSDNN}_{30 s}$, a partir dos valores de pico do exercício e recuperação pós-exercício, obtidas pela equação: $\sum[($ Área1+Área2+Área 3...)], onde cada área: [(Base maior + Base menor) x altura]/2. Foi considerado $30 \mathrm{~s}$ para altura obtendo-se 20 áreas, posteriormente somadas e obtido o valor da área total.

\section{Análise Estatística}

Foi utilizada estatística descritiva com determinação da média e desvio padrão das variáveis estudadas. Para verificar a normalidade e homogeneidade dos dados foi utilizado o teste de Shapiro Wilk e de Levene $(\mathrm{p}<0,05)$, respectivamente. Para comparar as variáveis em repouso, exercício e as áreas totais da VFC entre os tipos de recuperação utilizou-se o teste $\mathrm{T}$ pareado para dados paramétricos e o teste Wilcoxon para dados não paramétricos $(\mathrm{p}<0,05)$. O comportamento dos índices da VFC a cada 30 $s$ de recuperação foram comparados pela ANOVA Two-way de medidas repetidas seguido pelo post hoc de Bonferroni, com verificação dos dados quanto a violação da esfericidade pelo teste de Mauchly e a correção de Greenhouse-Geisser utilizada quando a esfericidade foi violada. $\mathrm{O}$ nível de significância adotado foi de $5 \%(\mathrm{p}<0,05)$. 


\section{Resultados}

A TABELA 1 apresenta os valores de média e desvio-padrão das variáveis em repouso e exercício.

Como esperado, não foram encontradas diferenças ( $p>0,05)$ para as variáveis analisadas em repouso e

RMSSD: Raiz quadrada da média das diferenças sucessivas ao quadrado entre $\mathrm{R}-\mathrm{R}$ adjacentes; SDNN: Desvio padráo de todos os intervalos R-R normais gravados em um intervalo de tempo. $\mathrm{VO}_{2}$ pico: pico do consumo do oxigênio.

A seta indica o final da recuperação ativa e passiva. que as voluntárias realizaram a recuperação pósexercício em condiçōes fisiológicas similares.

A FC não diferiu $(p>0,05)$ entre os tipos de recuperação ativa ou passiva em nenhum dos tempos da recuperação pós-exercício (FIGURA 1).

TABELA 1 -Média e desvio padrão das variáveis hemodinâmicas e autonômicas em repouso e exercício.

\begin{tabular}{lccc}
\hline & Ativa & Passiva & p valor \\
\hline Frequência cardíaca repouso (bpm) & $80,39 \pm 16,57$ & $80,94 \pm 16,90$ & 0,250 \\
LnRMSSD repouso (ms) & $42,32 \pm 35,15$ & $39,66 \pm 30,07$ & 0,558 \\
LnSDNN repouso (ms) & $57,97 \pm 30,26$ & $57,17 \pm 31,32$ & 0,759 \\
Frequência cardíaca pico (bpm) & $192,78 \pm 7,90$ & $192,89 \pm 7,72$ & 0,918 \\
Carga máxima (w) & $143,28 \pm 31,48$ & $144,67 \pm 26,25$ & 0,663 \\
$\mathbf{V O}_{\mathbf{2}}$ pico $\left(\mathbf{m l} \cdot \mathbf{k g} \cdot \mathbf{m i n}^{-1}\right)$ & $32,44 \pm 7,26$ & $32,50 \pm 6,37$ & 0,612 \\
\hline
\end{tabular}

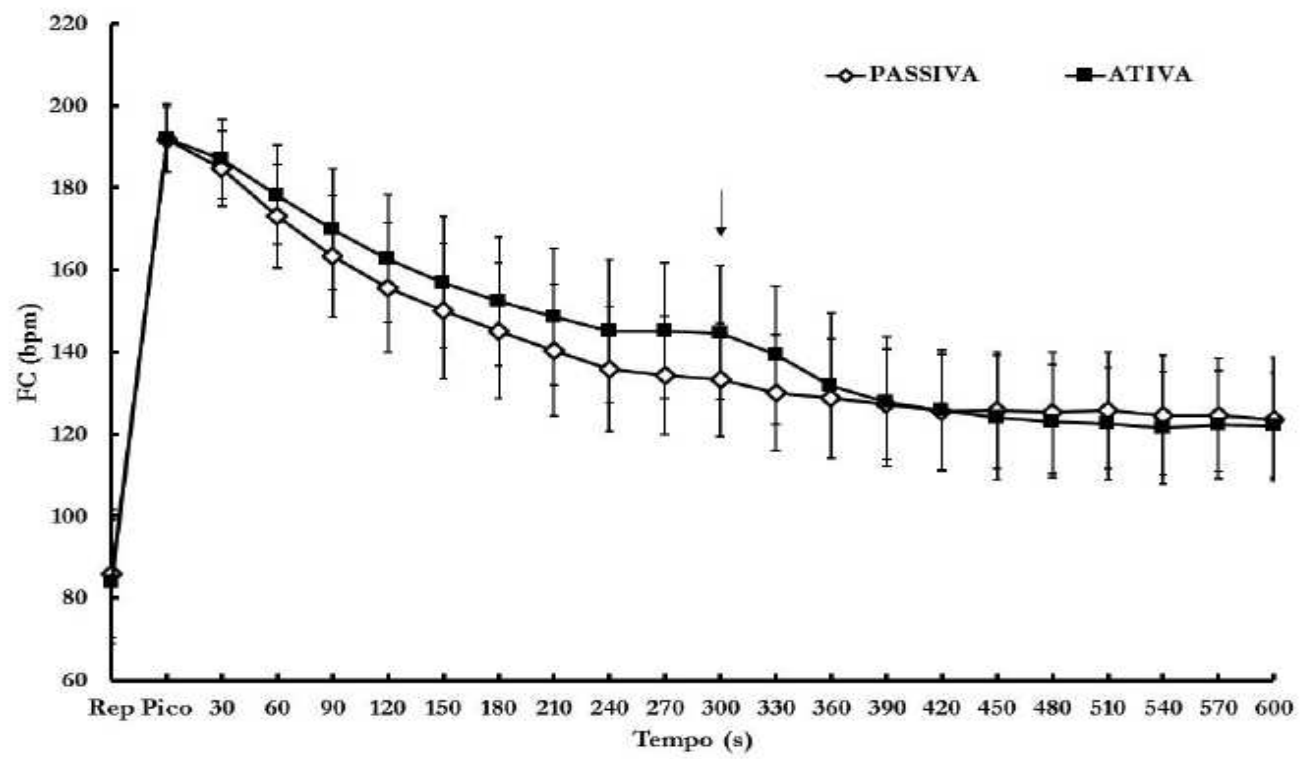

FIGURA 1 -Comportamento da FC no repouso, pico do exercício e a cada 30 s pós-exercício máximo.

A FIGURA 2 A e B apresenta o comportamento dos índices LnRMSSD e LnSDNN no repouso, pico do exercício e na recuperação analisados a cada $30 \mathrm{~s}$ durante os primeiros cinco min de recuperação ativa e passiva, e nos cinco min subsequentes de recuperação. Quanto ao tipo de recuperação, foi observado maiores valores apenas aos $180 \mathrm{~s}$ do índice LnRMSSD30 e aos 150 e 330 s do índice $\operatorname{LnSDNN}_{30 \text { s }}$ da recuperação ativa comparada a passiva, e menores valores aos 60 e $270 \mathrm{~s}$ do índice $\operatorname{LnSDNN}_{30 s}$ da recuperação ativa comparada a passiva.

Ao avaliar o comportamento da VFC, os índices $\operatorname{LnRMSSD}_{30 \mathrm{~s}}$ e $\operatorname{LnSDNN}_{30 \mathrm{~s}}$ ao final do exercício (A: $0,52 \pm 0,13-$ p: 0,016; P: 0,55 $\pm 0,25-$ p: 0,024;
/ A: $0,42 \pm 0,11-\mathrm{p}: 0,000 ; \mathrm{P}: 0,48 \pm 0,21 \mathrm{~ms}-\mathrm{p}$ : $0,001)$ estão reduzidos em relação ao repouso (A: $1,49 \pm 0,29 ; \mathrm{P}: 1,46 \pm 0,28 / \mathrm{A}: 1,70 \pm 0,21 ; \mathrm{P}: 1,69$ $\pm 0,20 \mathrm{~ms})$ e permaneceram inferiores ao final dos 5 min da recuperação (A: $0,53 \pm 0,12-p: 0,011 ; P$ : $0,45 \pm 0,14-p: 0,005 / A: 0,63 \pm 0,12-p: 0,000$; P: 0,68 $\pm 0,24 \mathrm{~ms}-\mathrm{p}: 0,006)$ e após esse período (A: 0,48 $\pm 0,12-\mathrm{p}: 0,021 ; \mathrm{P}: 0,55 \pm 0,23-\mathrm{p}: 0,017$ / A: $0,86 \pm 0,20-\mathrm{p}: 0,001 ; \mathrm{P}: 0,87 \pm 0,20 \mathrm{~ms}-\mathrm{p}$ : $0,002)$ sem diferença entre os tipos de recuperação. Entretanto, durante (aos 60, 90 e 150 s), e após a recuperação ativa, os valores do índice $\mathrm{LnSDNN}_{30 \text { s, }}$ que reflete a interação simpato-vagal, elevaram-se em relação ao pico do exercício $(\mathrm{p}<0,05)$ exceto aos 480 (p: 0,291), 510 (p: 0,149) e 600 s (p: 0,146), 
indicando retirada simpática, uma vez que o índice LnRMSSD, que reflete exclusivamente a atividade parassimpática, não retornou aos valores de repouso durante e após a recuperação (FIGURA 2).

Independentemente do tipo de recuperação não houve reativação vagal pós-exercício máximo em jovens não treinadas, contudo a recuperação ativa auxiliou no reestabelecimento da modulação autonômica cardíaca, reduzindo a janela de exposição aos riscos cardiovasculares pós-exercício, uma vez que, houve início da retirada simpática, apesar da média total da área sob a curva dos índices LnRMSSD $_{30 \mathrm{~s}}$ (A: 320,84 \pm 101,82; P: 332,16 \pm 177,91 ms - p: 0,299) e $\operatorname{LnSDNN}_{30 \mathrm{~s}}$ (A: 470,19 \pm $104,11$; P: $494,05 \pm 130,95 \mathrm{~ms}-\mathrm{p}: 0,386)$ não se diferirem $(p>0,05)$ entre os tipos de recuperação.

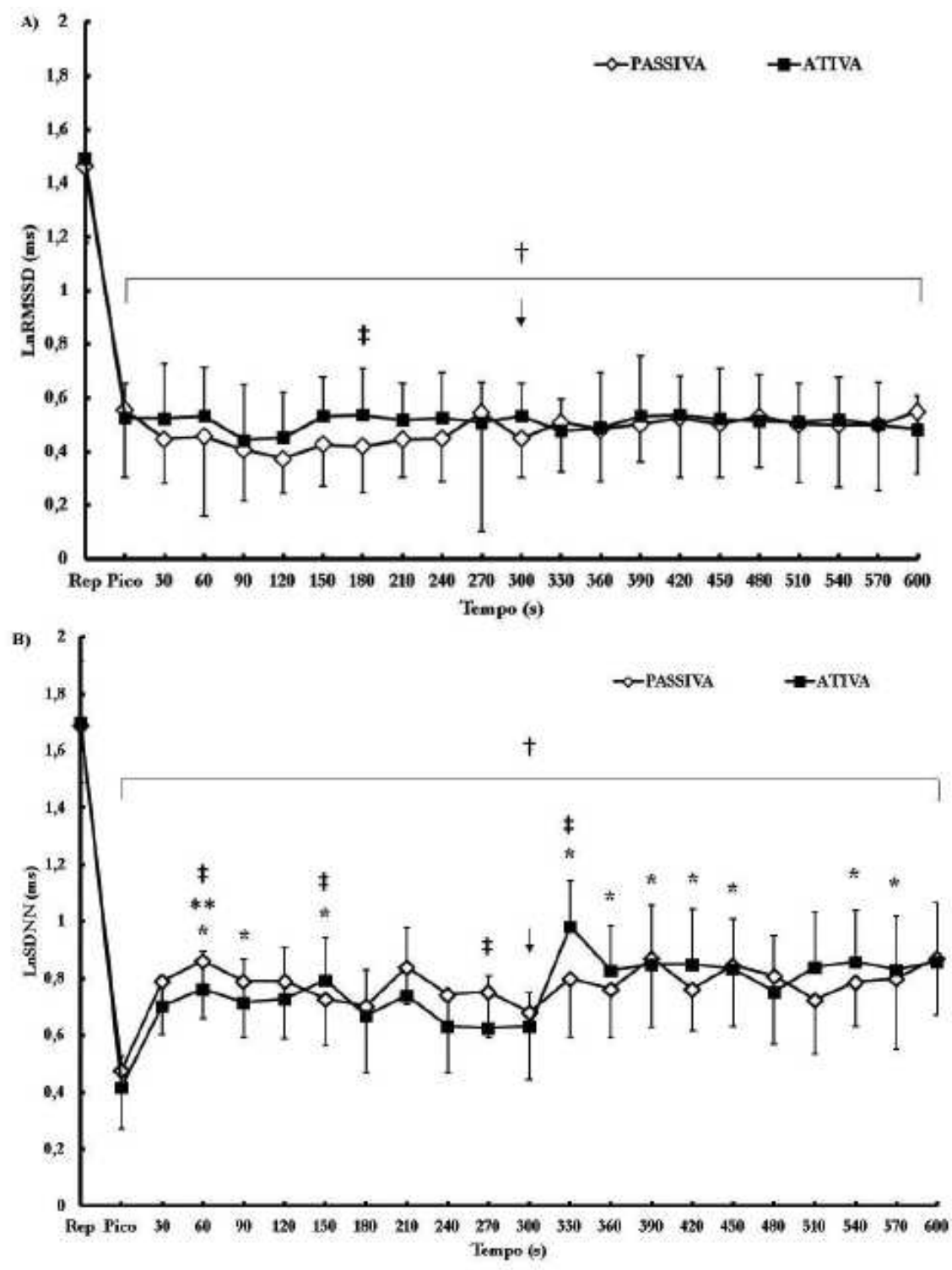

A seta indica o final

da recuperação ativa e passiva. ${ }^{\dagger} \mathrm{p}<0,05 \mathrm{em}$ relação ao repouso, ${ }^{*} \mathrm{p}<0,05$ em relação ao pico do exercício para recuperação ativa, ${ }^{* *} \mathrm{p}<0,05 \mathrm{em}$ relação ao pico do exercício para recuperação passiva e ${ }^{\ddagger} \mathrm{p}<0,05$ diferença entre a recuperação ativa e passiva.

FIGURA 2 -Comportamento do índice LnRMSSD (A) e LnSDNN (B) no repouso e LnRMSSD3o (A) e LnSDNN3o (B) no pico do exercício e pós-exercíco máximo

\section{Discussão}

Os principais resultados do presente estudo demonstram que pós-exercício máximo não houve reativação vagal, independentemente da recuperação ativa ou passiva, pois os índices $\operatorname{LnRMSSD}_{30 \mathrm{~s}}$ e $\operatorname{LnSDNN}_{30 \text { s }}$ após $10 \mathrm{~min}$ de recuperação permaneceram reduzidos em relação 
ao repouso em mulheres jovens não treinadas. Contudo, a recuperação ativa promoveu o início da retirada simpática, determinada pela elevaçáo do índice $\operatorname{LnSDNN}_{30 \text { s }}$ em relaçáo ao pico do exercício, contribuindo para o restabelecimento da modulação autonômica cardíaca.

A importância do presente estudo se dá por avaliar a influência do tipo de recuperação no restabelecimento da modulação autonômica cardíaca, e consequentemente na janela de exposição aos riscos cardiovasculares pós-exercício máximo, por meio de janelamentos de $30 \mathrm{~s}$ dos índices de VFC, em mulheres jovens saudáveis não treinadas. Uma vez que a recuperação pós-exercício é bastante estudada na avaliação de risco de mortalidade ${ }^{9,12}$, no acometimento de doenças cardiovasculares ${ }^{1,27}$, assim como, para avaliar a reativação vagal em homens jovens saudáveis ${ }^{6,17,28}$, fisicamente ativos $^{7,11}$, não treinados ${ }^{2,5,10,13}$ e em menor escala na comparação de tipos de recuperaçãoo ${ }^{14,16,19,29,30}$. Além disso, o fato de avaliar mulheres, que são pouco estudadas ${ }^{18,31,32}$, e apresentam maior proteção vagal cardíaca, devido a maior atividade vagal em repouso e restabelecimento vagal pós-exercício ${ }^{33}$, em relação aos homens, independente da aptidão aeróbia ${ }^{34}$. Sendo que, essas diferenças podem ser determinadas pelos hormônios ovarianos, como o estrógeno, que aumenta o tônus vasomotor, a integridade vascular e a sensibilidade barorreflexa ${ }^{35-38}$.

O exercício máximo resulta em elevação da atividade simpática e dos níveis sanguíneos de catecolaminas ${ }^{27,39}$ que contribuem para o atraso do restabelecimento vagal na recuperação pós-exercício ${ }^{40}$. Além disso, na recuperação, as respostas da VFC estão relacionadas à modalidade (caminhada, corrida e ciclismo $)^{17}$, duração e intensidade do exercício prévio $^{2}$, tipo de recuperação (ativa e passiva) ${ }^{14,16}$, população estudada (jovens e idosos) ${ }^{28}$ e tipo de treinamento (aeróbio) ${ }^{11,32}$.

Alguns estudos sugerem que há reativação vagal apenas pós-exercício submáximo, como verificado na recuperação passiva pelo aumento do RMSSD em jovens não treinados ${ }^{5,41}$, e em fisicamente ativos $^{6}$ com retorno aos valores de repouso após 65 min, sendo mais lenta após exercício vigoroso ${ }^{41}$, e influenciada pela aptidão aeróbia, pois indivíduos com alta e baixa aptidão aeróbia apresentaram reativação vagal aproximadamente aos 9,5 e $25 \mathrm{~min}$, respectivamente ${ }^{6}$. Por outro lado, em estudos com menor tempo de análise (10 e $15 \mathrm{~min}$ ) não houve reativação vagal ${ }^{13,32}$.

No presente estudo, os valores do índice
LnRMSSD $_{30 \mathrm{~s}}$ mantiveram-se reduzidos nos $10 \mathrm{~min}$ após exercício em relação ao repouso e ao final do exercício máximo demonstrando a não reativação vagal. Corroborando outros estudos, que avaliaram a recuperação passiva em mulheres treinadas e não treinadas ${ }^{31}$, homens fisicamente ativos ${ }^{7}$, indivíduos sedentários ${ }^{11} \mathrm{e}$ a recuperação ativa em jovens não treinados com diferentes índices de massa corporal pós-exercício máximo em curtos períodos de recuperação.

Reativação vagal foi observada em jovens eutróficos ${ }^{17}$ e treinados aerobiamente ${ }^{11}$ após recuperação passiva de 3 e 5 min pós-exercício máximo. Entretanto, sem retorno aos valores do índice $\mathrm{RMSSD}_{30 \mathrm{~s}}$ pré-exercício, além disso, as populações estudadas diferem do presente estudo, uma vez que apresentam maior aptidão aeróbia e que está associada à melhor recuperação autonômica cardíaca ${ }^{11,17,28}$.

Os valores do índice $\mathrm{LnSDNN}_{30 \mathrm{~s}}$ mantiveramse reduzidos em relação ao repouso durante a recuperação ativa e passiva, contudo nos cinco min após a recuperação ativa, o $\operatorname{LnSDNN}_{30 s}$ elevou-se significativamente, o que indica a retirada simpática, e se assemelha aos resultados de jovens fisicamente ativos ${ }^{7}$ e em jovens não treinados na recuperação passiva e ativa, respectivamente. Dessa forma, o restabelecimento da modulação autonômica cardíaca, após exercício máximo, parece estar associado à retirada inicial da atuação da atividade simpática.

Divergindo dos nossos resultados, não foi constatada influência do tipo de recuperação ativa e passiva nos índices LnSDNN, LnRMSSD, LnHF (u.n.) e $\operatorname{LnLF}$ (u.n.), analisados nos 15 e $30 \mathrm{~min}$ pós-exercício submáximo ${ }^{14}$ e nos índices SDNN e RMSSD, analisados a cada 15 min durante 90 min pós-exercício máximo ${ }^{16}$, o que também pode ter ocorrido devido a análise da VFC não ter sido realizada pelo janelamento de $30 \mathrm{~s}$, que é o método mais adequado para identificar alterações da VFC pós-exercício ${ }^{10}$.

$\mathrm{Na}$ recuperação ativa, além do comando central sobre as respostas cardiovasculares, a contração dos músculos gastrocnêmios proporciona maior retorno venoso sanguíneo ao coração, e resulta em uma gradual redução da pressão arterial, da taxa de enchimento ventricular, volume sistólico e débito cardíaco ${ }^{19,42}$, e em menor risco cardiovascular pós-exercício ${ }^{19,20}$. Devido a redução gradual das variáveis hemodinâmicas, a síncope comum quando o exercício máximo é interrompido abruptamente é 
evitada $^{27}$. Entretanto, na recuperação passiva, para suprir a diminuição abrupta do retorno venoso, ocorre vasoconstrição ${ }^{19,42}$ que em conjunto com os mecanismos reflexos desativados (barorreceptores arteriais), podem aumentar a ativação do sistema nervoso simpático ${ }^{43}$ e explicar a ausência da retirada simpática após a recuperação passiva do presente estudo.

Além da influência no sistema cardiovascular ${ }^{19,42}$, o tipo de recuperação influência na concentração dos metabólitos pós-exercício ${ }^{16}$. A recuperação ativa proporciona maiores níveis de hormônios circulantes, tais como cortisol, testosterona e hormônio do crescimento ${ }^{30}$, acelera a remoção de lactato, e reduz os níveis de $\mathrm{H}^{+}$e $\mathrm{PO}_{2}{ }^{16,29}$ comparada à recuperação passiva, devido ao maior fluxo sanguíneo para os músculos ativos ${ }^{44}$.

Adicionalmente, pós-exercício máximo os mecanismos envolvidos na recuperação são restabelecidos em diferentes momentos ${ }^{15,16}$, ocorrendo primeiramente a reestabilização metabólica pelo lactato sanguíneo, reorganização simpato-vagal, seguido da restauração vagal completa, sendo que os índices SDNN e LF (reflete atividade simpática) apresentam restabelecimento anterior (60 min) aos índices RMSSD e HF - (reflete atividade parassimpática) $(70 \mathrm{~min})^{15}$, o que corrobora o presente estudo, uma vez que, o restabelecimento do índice relacionado a atividade simpática antecedeu as respostas de reativação vagal na reorganização da modulação autonômica cardíaca.

A divergência entre os estudos pode ser devida a população estudada, sexo, tipo e tempo, tanto de exercício quanto de recuperação, e tempo de análise após a recuperação. E, apesar das avaliaçôes terem ocorrido na fase folicular do ciclo menstrual, os níveis hormonais femininos não foram mensurados, sendo esta uma das limitações do presente estudo.

Em suma, independentemente do tipo de recuperação, ativa ou passiva, não houve reativação vagal após 10 min de recuperação pós-exercício máximo em mulheres jovens não treinadas. Entretanto, a recuperação ativa auxiliou na retirada da atividade simpática determinada pela elevação do índice $\operatorname{LnSDNN}_{30 \text { s }}$, proporcionando assim, o restabelecimento da modulação autonômica cardíaca, reduzindo a janela de exposição aos riscos cardiovasculares pós-exercício.

\title{
Conflito de interesse
}

Os autores declaram não haver conflito de interesses.

\begin{abstract}
Active recovery improve sympathetic withdrawal post maximum exercise in untrained young women

The aim this study was to assess the heart rate variability (HRV) after active recovery of a maximal exercise in untrained young. Nine normal weight women $\left(25.45 \pm 5.41\right.$ years old, $22.23 \pm 2.03 \mathrm{~kg} \mathrm{~m}^{-2} ; 28.13 \pm 5.0 \%$ body fat) were evaluated at rest, exercise and at recovery post- maximum exercise in cycle ergometer performed active (A) or passive (P) form, with the HRV index (SDNN and RMSSD) assessed during the 5 min of recovery and $5 \mathrm{~min}$ later at every $30 \mathrm{~s}$. The $\operatorname{LnRMSSD}_{30 \mathrm{~s}}$ and $\operatorname{LnSDNN}_{30 \mathrm{~s}}$ index in the end of the exercise $(A$ : $0.52 \pm 0.15 ; P: 0.55 \pm 0.24$ / A: $0.42 \pm 0.12 ; P: 0.48 \pm 0.18 \mathrm{~ms})$ reduced in relation to rest $(A: 1.49 \pm 0.39 ;$ $P: 1.46 \pm 0.41 / A: 1.70 \pm 0.26 ; P: 1.69 \pm 0.27 \mathrm{~ms})$, and remained reduced $(p<0.05)$ at the end of the first $5 \mathrm{~min}$ of recovery $(A: 0.53 \pm 0.16, P: 0.45 \pm 0.18 / A: 0.63 \pm 0.21 ; P: 0.68 \pm 0.29 \mathrm{~ms})$ and after this time (A: $0.48 \pm 0.20 ; P: 0.55 \pm 0.24$ / A: $0.86 \pm 0.27 ; P: 0.87 \pm 0.26 \mathrm{~ms})$. However, after active recovery, $\operatorname{LnSDNN}_{30 \mathrm{~s}}$ index increased in relation to the end of exercise $(p<0.05)$. In conclusion, regardless of recovery type, there was no vagal reactivation in $10 \mathrm{~min}$ after a maximal exercise in untrained young women, however the active recovery favored in restoring cardiac autonomic modulation through the sympathetic withdrawal.
\end{abstract}

KEYWORDS: Autonomic Nervous System; Heart rate variability; Vagal reactivation. 


\section{Referências}

1. Kannankeril PJ, Le FK, Kadish AH, Goldberger JJ. Parasympathetic effects on heart rate recovery after exercise. J Invest Med. 2004;52(6):394-401.

2. Peçanha T, Prodel E, Bartels R, Nasario-Junior O, Paula R, Silva L, et al. 24-h cardiac autonomic profile after exercise in sedentary subjects. Int J Sports Med. 2014;35(03):245-52.

3. Albert CM, Mittleman MA, Chae CU, Lee IM, Hennekens CH, Manson JE. Triggering of sudden death from cardiac causes by vigorous exertion. N Engl J Med. 2000;343(19):1355-61.

4. Imai K, Sato H, Hori M, Kusuoka H, Ozaki H, Yokoyama H, et al. Vagally mediated heart rate recovery after exercise is accelerated in athletes but blunted in patients with chronic heart failure. J Am Coll Cardiol. 1994;24(6):1529-35.

5. Javorka M, Zila I, Balharek T, Javorka K. Heart rate recovery after exercise: relations to heart rate variability and complexity. Braz J Med Biol Res. 2002;35(8):991-00.

6. Oliveira TP, Lima JRP. Post-exercise cardiac full vagal reactivation: initial proposal and influence of physical training. J Appl Phys. 2012;15(6):103-12.

7. Oliveira TP, Alvarenga Mattos R, Silva RBF, Rezende RA, Lima JRP. Absence of parasympathetic reactivation after maximal exercise. Clin Physiol Funct Imaging. 2013;33(2):143-49.

8. Araújo JA, Tricot GK, Arsa G, Queiroz MG, Santos KM, Dias ARL, et al. Blood pressure and cardiac autonomic modulation at rest, during exercise and recovery time in the young overweight. Motriz. 2016;22(1):27-34.

9. Cole C, Blackstone E, Pashkow F, Snader C, Lauer M. Heart rate recovery immediately after exercise as a predictor of mortality. N Engl J Med. 1999;18(2):1351-57.

10. Goldberger JJ, Le FK, Lahiri M, Kannankeril PJ, Ng J, Kadish AH. Assessment of parasympathetic reactivation after exercise. Am J Physiol Heart Circ Physiol. 2006;290(6):H2446-52.

11. Guerra ZF, Peçanha T, Moreira DN, Silva LP, Laterza MC, Nakamura FY, et al. Effects of load and type of physical training on resting and postexercise cardiac autonomic control. Clin Physiol Funct Imaging. 2014;34(2):114-20.

12. Nishime EO, Cole CR, Blackstone EH, Pashkow FJ, Lauer MS. Heart rate recovery and treadmill exercise score as predictors of mortality in patients referred for exercise ECG. Jama. 2000;284(11):1392-8.

13. Barak OF, Jakovljevic D, Popadic GJZ, Ovcin ZB, Brodie D, Grujic N. Heart rate variability before and after cycle exercise in relation to different body positions. J Sports Sci Med. 2010;9:176-82.

14. Soares AH, Oliveira TP, Cavalcante BR, Farah BQ, Lima AH, Cucato GG, et al. Effects of active recovery on autonomic and haemodynamic responses after aerobic exercise. Clin Physiol Funct Imaging. 2017;37(1):62-67.

15. Hoshi RA, Vanderlei LCM, Godoy MF, Bastos FdN, Netto J, Pastre CM. Temporal sequence of recovery-related events following maximal exercise assessed by heart rate variability and blood lactate concentration. Clin Physiol Funct Imaging. 2017;37(5):536-543.

16. Bastos FN, Vanderlei LCM, Nakamura FY, Bertollo M, Godoy MF, Hoshi RA, et al. Effects of cold water immersion and active recovery on post-exercise heart rate variability. Int J Sports Med. 2012;33(11):873-79.

17. Cunha FA, Midgley AW, Gonçalves T, Soares PP, Farinatti P. Parasympathetic reactivation after maximal CPET depends on exercise modality and resting vagal activity in healthy men. SpringerPlus. 2015;4(100):1-9.

18. Journeay S, Reardon FD, McInnis NH, Kenny GP. Nonthermoregulatory control of cutaneous vascular conductance and sweating during recovery from dynamic exercise in women. J Appl Physiol. 2005;99(5):1816-21.

19. Carter R, Watenpaugh DE, Wasmund WL, Wasmund SL, Smith ML. Muscle pump and central command during recovery from exercise in humans. J Appl Physiol. 1999;87(4):1463-69.

20. Medicine ACoS. Diretrizes do ACSM para os testes de esforço e sua prescrição. Rio de Janeiro, RJ: Guanabara Koogan; 2007.

21. Foster-Powell K, Holt SH, Brand-Miller JC. International table of glycemic index and glycemic load values: 2002. Am J Clin Nutr. 2002;76(1):5-56.

22. Sociedade Brasileira de C, Sociedade Brasileira de H, Sociedade Brasileira de N. [VI Brazilian Guidelines on Hypertension]. Arq Bras Cardiol. 2010;95(1 Suppl):1-51.

23. Fronchetti L. Limiar de variabilidade da freqüência cardíaca em diferentes protocolos ergométricos. Florianópolis: Universidade do Estado de Santa Catarina; 2008.

24. Kuipers H, Verstappen F, Keizer H, Geurten P, Van Kranenburg G. Variability of aerobic performance in the laboratory and its physiologic correlates. Int J Sports Medicine. 1985;6(04):197-01.

25. Borg GA. Psychophysical bases of perceived exertion. Med Sci Sports Exerc. 1982;14(5):377-81.

36o • Rev Bras Educ Fís Esporte, (São Paulo) 2019 Jul-Set; 33(3):353-61 
26. Howley ET, Bassett DR, Welch HG. Criteria for maximal oxygen uptake: review and commentary. Med Sci Sports Exer. 1995;27(9):1292-01.

27. Peçanha T, Silva-Júnior ND, Forjaz CLdM. Heart rate recovery: autonomic determinants, methods of assessment and association with mortality and cardiovascular diseases. Clin Physiol Funct Imaging. 2014;34(5):327-39.

28. Trevizani GA, Benchimol-Barbosa PR, Nadal J. Effects of age and aerobic fitness on heart rate recovery in adult men. Arq Bras Cardiol. 2012;99(3):802-10.

29. Wahl P, Mathes S, Köhler K, Achtzehn S, Bloch W, Mester J. Effects of active vs. passive recovery during Wingate-based training on the acute hormonal, metabolic and psychological response. Growth Horm IGF Res. 2013;23(6):201-08.

30. Wahl P, Mathes S, Achtzehn S, Bloch W, Mester J. Active vs. passive recovery during high-intensity training influences hormonal response. Int J Sports Med. 2014;35(07):583-89.

31. Du N, Bai S, Oguri K, Kato Y, Matsumoto I, Kawase H, et al. Heart rate recovery after exercise and neural regulation of heart rate variability in 30-40 year old female marathon runners. J Sports Sci Med. 2005;4(1):9-17.

32. Paschoal MA, Pinheiro TT, Brigliador GM, Caruso TMA, de Lima LNG. Efeito do treinamento físico aeróbio sobre a reativação vagal cardíaca em jovens sedentárias. Rev Bras Ativ Fis e Saúde. 2013;17(5):403-13.

33. Antelmi I, Chuang EY, Grupi CJ, Latorre MRD. Heart rate recovery after treadmill electrocardiographic exercise stress test and 24-hour heart rate variability in healthy individuals. Arq Bras Cardiol. 2008;90(6):413-18.

34. Kappus RM, Ranadive SM, Yan H, Lane-Cordova AD, Cook MD, Sun P, et al. Sex differences in autonomic function following maximal exercise. Biol Sex Differ. 2015;6(28):1-8.

35. Mckinley PS, King AR, Shapiro PA, Slavov I, Fang Y, Chen IS, et al. The impact of menstrual cycle phase on cardiac autonomic regulation. Psychophysiology. 2009;46(4):904-11.

36. Minson CT, Halliwill JR, Young TM, Joyner MJ. Influence of the menstrual cycle on sympathetic activity, baroreflex sensitivity, and vascular transduction in young women. Circulation. 2000;101(8):862-68.

37. Mohamed MK, El-Mas MM, Abdel-Rahman AA. Estrogen enhancement of baroreflex sensitivity is centrally mediated. Am J Physiol Regul Integr Comp Physiol. 1999;276(4):R1030-37.

38. Santos RL, Silva F, Ribeiro RF. Sex hormones in the cardiovascular system. Horm Mol Biol Clin Invest. 2014;18(2):89103.

39. Pierpont GL, Stolpman DR, Gornick CC. Heart rate recovery post-exercise as an index of parasympathetic activity. J Autonom Nervous System. 2000;80(3):169-74.

40. Coote JH. Recovery of heart rate following intense dynamic exercise. Exp Physiol. 2010;95(3):431-40.

41. Gladwell V, Sandercock G, Birch S. Cardiac vagal activity following three intensities of exercise in humans. Clin Physiol Funct Imaging. 2010;30(1):17-22.

42. Crisafulli A, Orru V, Melis F, Tocco F, Concu A. Hemodynamics during active and passive recovery from a single bout of supramaximal exercise. Eur J Appl Physiol. 2003;89(2):209-16.

43. La Rovere MT, Pinna GD, Raczak G. Baroreflex sensitivity: measurement and clinical implications. Ann Noninvasive Electrocardiol. 2008;13(2):191-07.

44. Ahmaidi S, Granier P, Taoutaou Z, Mercier J, Dubouchaud H. Effects of active recovery on plasma lactate and anaerobic power following repeated intensive exercise. Med Sci Sports Exerc. 1996;28(4):450-56.

ENDERECOO DE CORRESPONDÊNCIA: Lucieli Teresa Cambri

Universidade Federal de Mato Grosso, UFMT Av. Fernando Corrêa da Costa, 2367 - Bairro Boa Esperança

Cuiabá - MT - BRASIL CEP: 78060-900

E-mail: lucambri@yahoo.com.br
Submetido: 10/08/2016

Revisado: $15 / 11 / 2016$

Aceito: 13/02/2017 